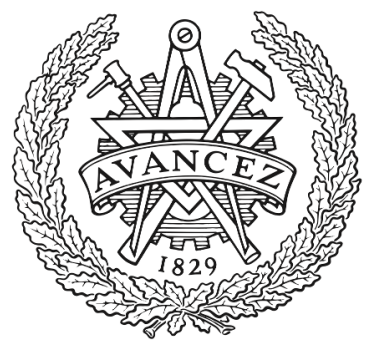

CHALMERS

UNIVERSITY OF TECHNOLOGY

\title{
Loads at the implant-prosthesis interface during free and aided ambulation in osseointegrated transfemoral prostheses
}

Downloaded from: https://research.chalmers.se, 2023-04-26 07:17 UTC

Citation for the original published paper (version of record):

Thesleff, A., Häggström, E., Tranberg, R. et al (2020). Loads at the implant-prosthesis interface during free and aided ambulation in osseointegrated transfemoral prostheses. IEEE Transactions on Medical Robotics and Bionics, 2(3): 497-505. http://dx.doi.org/10.1109/TMRB.2020.3002259

N.B. When citing this work, cite the original published paper. 


\title{
Loads at the implant-prosthesis interface during free and aided ambulation in osseointegrated transfemoral prostheses
}

\author{
Alexander Thesleff, Student Member, IEEE, Eva Häggström, Roy Tranberg, Roland Zügner, Anders \\ Palmquist, Max Ortiz-Catalan, Member, IEEE
}

\begin{abstract}
Bone-anchored attachment of amputation limb prostheses is increasingly becoming a clinically accepted alternative to conventional socket suspension. The direct transfer of loads demands that the percutaneous implant system and the residual bone withstand all forces and moments transferred from the prosthesis. This study presents load measurements recorded at the bone-anchored attachment in 20 individuals with unilateral transfemoral amputation performing the everyday ambulatory activities: level ground walking, stairs ascent/descent and slope ascent/descent. Mean peak values for the sample populations across activities ranged from 498-684 $\mathrm{N}$ for the resultant force, 26.5-39.8 Nm for the bending moment, and 3.1-5.5 Nm for the longitudinal moment. Significant differences with respect to level walking were found for the resultant force during stairs ascent, (higher, $p=0.002$ ), and stairs descent, (lower, $p=0.005$ ). Using a crutch reduced the peak resultant forces and the peak bending moments with averages ranging from 5.5-12.6 \% and 13.2-15.6 $\%$, respectively. Large inter-participant variations were observed and no single activity resulted in consistently higher loading of the bone-anchored attachment across the participants. Results from this study can guide future development of percutaneous osseointegrated implant systems for limb prostheses and their rehabilitation protocols.
\end{abstract}

Index Terms-Bone-anchored prosthesis, Daily walking activities, Load measurement, Osseointegration, Transfemoral amputation

\section{INTRODUCTION}

$\mathrm{T}$ HE conventional way of attaching a limb prosthesis to the body is by means of a socket that compresses the soft tissue over the residual limb stump. This attachment method frequently causes problems such as discomfort, dermatologic complications, poor load transfer and retention, and limited range of movement [1]-[5]. It is to address these problems that percutaneous bone-anchored implant systems for direct skeletal attachment of limb prostheses have been developed. A titanium implant is surgically inserted intramedullary into the bone of the residual limb. During a healing period, bone tissue is formed

Research supported by research grants from the Swedish Foundation for Strategic Research (SSF), the Swedish Agency for Innovation (VINNOVA), the Promobilia Foundation, and the IngaBritt and Arne Lundbergs Foundation (Corresponding author: Max Ortiz-Catalan, maxo@chalmers.se)

A. Thesleff is with the Center for Bionics and Pain Research, Gothenburg, Sweden, with the Department of Electrical Engineering, Chalmers University of Technology, Gothenburg, Sweden, and also with Integrum AB, Mölndal Sweden. in direct contact with the implant creating a strong mechanical bond between the two [6], [7]. The distal end of the implant extending percutaneously from the end of the residuum allows for direct attachment of the prosthesis, thus eliminating the need for a socket.

The benefits with direct skeletal attachment are multifold, with added sensory feedback through osseoperception [8], [9], more efficient gait by reducing the metabolic cost of ambulation compared with a socket connection [10], and reduced sores and skin irritation [10], all leading to a general increased prosthetic use among the patients [11].

The first successful implementation of direct skeletal attachment for amputation prostheses took place in Sweden in 1990 [12] and following subsequent development from this original implant system, a standardized treatment was introduced in 1999 with the OPRA (Osseointegrated Prostheses for the Rehabilitation of Amputees) implant system (Integrum AB, Mölndal, Sweden) [12]. The OPRA implant system consists of a Fixture, an Abutment, and an Abutment Screw. The screw-shaped Fixture is fully implanted into the medullary cavity and allowed to osseointegrate in the residual bone. The Abutment is press-fitted into the Fixture and protrudes through the skin to the exterior of the body. The connection between the Fixture and the Abutment is further stabilized by a preload provided by the Abutment Screw (Fig. 1 (a)).

Since its first introduction, the technology has become more mature and clinically accepted [12], and prospective clinical trials of 51 patients (55 implants) have shown increased quality of life, physical function, and overall well-being at two [10] and five year [11] follow-ups. However, mechanical complications requiring replacement of Abutment and Abutment Screws over time has been raised as a concern [11], [13].

A consequence of the more efficient load transfer between the prosthesis and the skeleton is that increased mechanical demand is placed both on the bone and the implant system. Excessive forces and moments applied to the prosthesis, for example due to a fall [14], could potentially cause damage to

E. Häggström, R. Tranberg and R. Zügner are with Department of Orthopaedics, Institute of Clinical Sciences, Sahlgrenska Academy, Gothenburg University, Mölndal, Sweden.

A. Palmquist is with Department of Biomaterials, Gothenburg University, Gothenburg, Sweden.

M. Ortiz-Catalan is with Center for Bionics and Pain Research, Gothenburg, Sweden, with the Department of Electrical Engineering, Chalmers University of Technology, Gothenburg, Sweden, with Sahlgrenska University Hospital Mölndal, Sweden, and also with the Department of Orthopaedics, Institute of Clinical Sciences, Sahlgrenska Academy, University of Gothenburg, Sweden. 
the bone or the implant system. To avoid this, a safety device is mounted between the prosthesis and the implant system, unlocking the connection in case of excessive flexional or torsional loading at predefined thresholds. The thresholds are determined so that the safety mechanism only releases during loading that would otherwise put the implant or the bone at risk.

Forces and moments under the thresholds, generated from daily life activities (walking, standing, biking etc.), are transferred to the implant system and could potentially lead to fatigue of the implant or the bone if stress levels and the number of load cycles exceed what respective material can withstand. In order to understand whether this is a risk, accurate information of the maximum load state generated from everyday life activities is needed. In this regard, much of the previously published research has been limited to single-subject case studies [15], [16], or studies with small study samples [17]-[20], most of them only considering the activity of walking on level ground. Additionally, they have typically reported peak magnitude of individual force and moment components separately, possibly representing different instances in time. Therefore, making it impossible to discern the maximum load state experienced by the implant since the resultants from several force and moment component cannot be calculated. The primary aim of this study was therefore to characterize the maximum load state at the bone-anchored implant during daily life ambulatory activities.

One way to reduce the risk of mechanical failures would be to use a walking aid during certain activities to decrease the loading of the implant and the residual bone. The secondary aim of the study was to quantify the effectiveness of such measure in terms of reducing the maximum loads during every day ambulatory activities. This information is largely lacking in published research apart from a case study examining this effect in one subject and during level ground walking [21].

Bone-anchored attachment of amputation prostheses has not yet reached the maturity level of endo-prosthetic solutions such as hip and knee replacements. Standardized protocols for mechanical testing of the bone-anchored attachments have not yet been developed. The third aim of this study was therefore to provide clinically relevant input for future development of standardized test methods for fatigue testing of percutaneous implant for anchoring of limb prostheses.

To meet these objectives, direct load measurements were collected using a load cell placed between the prosthetic kneejoint and the Abutment in 20 individuals with unilateral transfemoral amputation treated with the OPRA Implant System while performing a number of everyday ambulatory activities.

\section{METHOD}

Inclusion criteria for participation was unilateral transfemoral amputation treated with a bone-anchored prosthetic attachment since at least two years prior to enrollment and with the ability to walk without any walking aids indoors. A load cell (iPECS Lab, College Park Industries, USA) was attached to the Abutment via a coupling device, and inclusion required at least $8.5 \mathrm{~cm}$ distance between the coupling

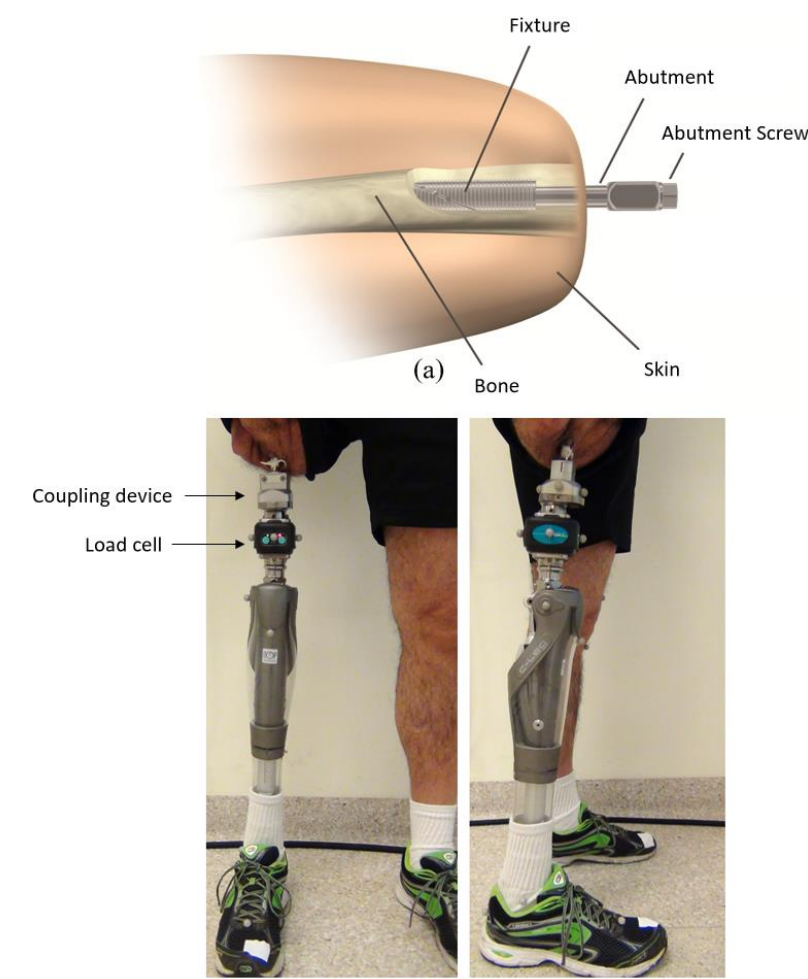

(b)

(c)

Fig. 1. (a) Schematic illustration of OPRATM Implant System. (b) Anterior view of subject equipped with the adjusted prosthesis with the loadcell attached to the Abutment with a coupling device prior to load measurements. (c) Lateral view.

device and the prosthetic knee joint in order to achieve an alignment close to the original. The research was conducted in accordance with the Declaration of Helsinki $\left(64^{\text {th }}\right.$ version 2013) and ethical approval was granted by the regional Swedish Ethical Committee (EPN/Gothenburg Dnr 130-09).

A total of 20 subjects were enrolled in the study. The subjects were selected as consecutive subjects visiting the Center of Orthopaedic Osseointegration (COO), fulfilling the inclusion criteria and agreeing to participate in the study. Informed consent was given by each subject prior to enrollment. Patients were excluded if they experienced pain during load bearing or had other disabilities affecting their walking ability. The measurements were acquired between February 2014 and April 2016 at Lundberglaboratoriet for orthopaedic research at Sahlgrenska University Hospital in conjunction with a followup visit to $\mathrm{COO}$, or when attending for other reasons. Both institutions are located in Gothenburg, Sweden. The demographics of the enrolled subjects and the performed daily life ambulatory activities are listed in Table I. 


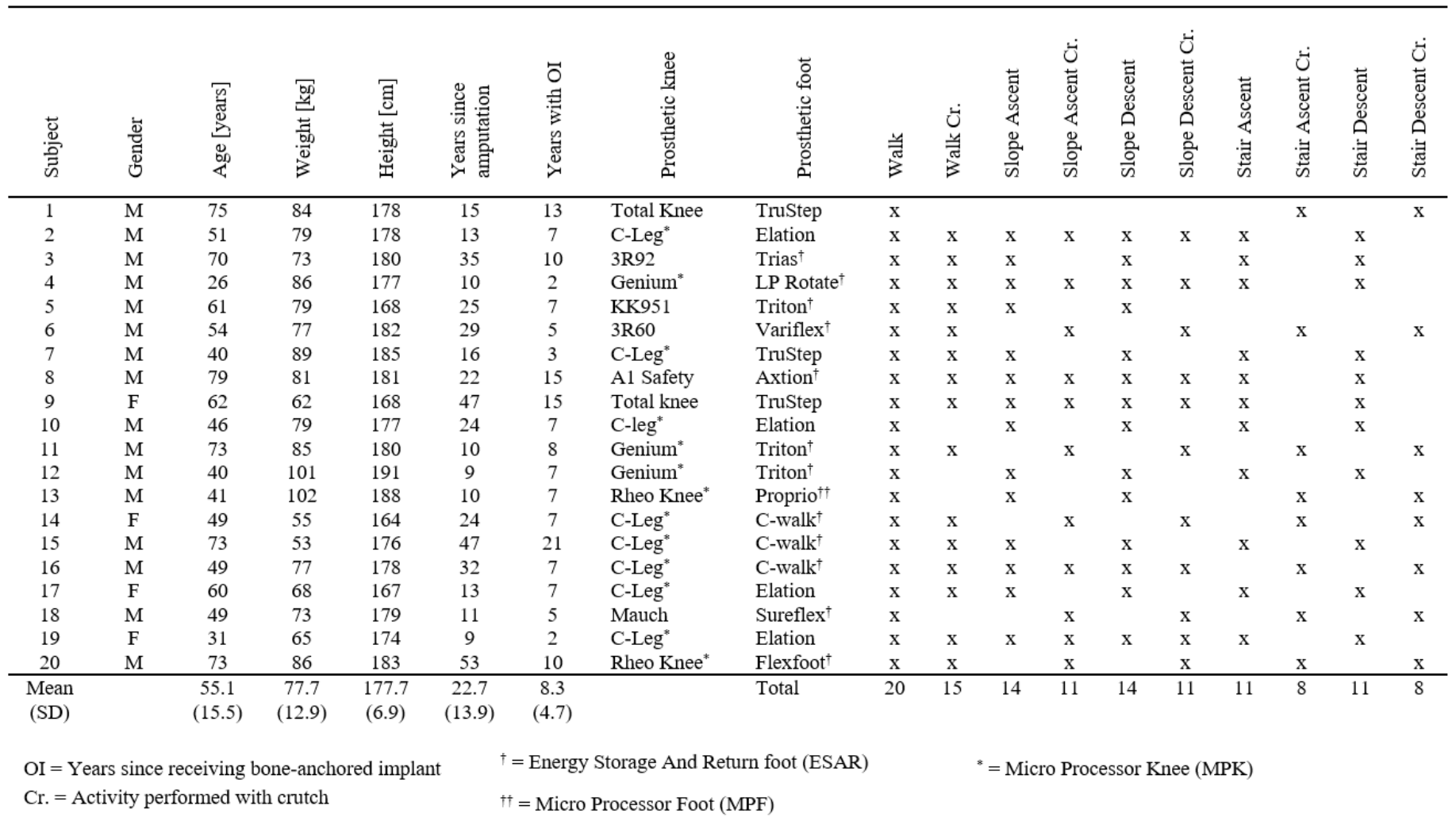

\section{A. Protocol}

Each subject used their own prosthesis during the load measurements. At the beginning of each session, the prosthesis was adapted by replacement of the safety device with the load cell. The load cell had 6 DoF (degrees of freedom) and a load range of $\pm 2670 \mathrm{~N} / \pm 282 \mathrm{Nm}$ ( $1 \%$ full scale accuracy) for the force and moment components in the transverse plane and $\pm 3560 \mathrm{~N} / \pm 45 \mathrm{Nm}(1.5 \%$ full scale accuracy) for the longitudinal axis. The same type of load cell has previously been used in related research [19], [22]-[25]. The load cell was aligned so that the z-direction of the load cell coincided with the longitudinal direction of the Abutment, and the y-direction of the load cell coincided with the anterior direction. Adjustments were made to ensure that the position of the prosthetic knee joint was at similar level as in the normal condition (maximum vertical deviation between original and adapted position of the knee-joint among all subjects was $3 \mathrm{~cm}$ ). All adjustments and modifications to the prosthesis were made by a certified prosthetist. Prior to donning the prosthesis, the load cell was zeroed. When the prosthesis was donned, the load cell center was located at a distance, $\mathrm{d}=133 \mathrm{~mm}$, distal to the distal interface between the Fixture and the Abutment. Fig. 1 (b) and (c) show an adjusted prosthesis equipped with the load cell.

The load measurements were recorded at a frequency of 240
$\mathrm{Hz}$ and streamed wirelessly to a laptop and stored in a text-file format. Prior to the first recording of the first activity (level walking), the subjects were asked to walk 5-10 times, at their self-selected speed through the measurement area $(6 \mathrm{~m} \times 2 \mathrm{~m})$ to familiarize themselves with the experimental setup.

The investigated daily life activities were, level walking, ascending/descending stairs and ascending/descending a slope. To maximize the relevance of the measurements all subjects were asked to perform each activity in the same manner as they normally would do it. Therefore, for subjects that normally used a walking aid when walking long distances, load measurements from level ground walking were recorded both with and without a crutch (their own) in the hand at the opposite side of the prosthesis. Similarly, for subjects who never used a crutch to perform an activity in their everyday life, no measurements were recorded of them using a crutch for that activity. Subjects were free to skip any activity which they did not feel comfortable performing.

The level walking activities were performed on level ground in a gait lab (6 m x $2 \mathrm{~m}$ measurement area). Stair ascent and stair descent was performed at a staircase consisting of $11 \mathrm{steps}$, (height $15.5 \mathrm{~cm}$, depth $35 \mathrm{~cm}$ ), with polished stone surface and access to a handrail which was optional to use. Slope ascent and slope descent was recorded in a $19.5 \mathrm{~m}$ walkway ( $2.5 \mathrm{~m}$ wide) at a $7.3^{\circ}$ angle on concrete ground with anti-slip stripes. Load data from at least two trials for each of the performed activities were recorded for each subject. 


\section{B. Data Processing}

The raw load data was processed and analyzed using custom scripts in Matlab 2018b (MathWorks Inc., Natick, MA, USA). The three force components were used to calculate the resultant force $\left(\mathrm{F}_{\mathrm{Res}}\right)$, and the anterior- and medio-lateral moment components $\left(\mathrm{M}_{\mathrm{A}}\right.$ and $\left.\mathrm{M}_{\mathrm{ML}}\right)$ were used to calculate the bending moment $\left(\mathrm{M}_{\mathrm{B}}\right)$ for each instance in time. With a rigid body assumption of the Abutment and the connection to the load-cell, the only components that differ between the load cell center and the distal end of the Fixture are the $\mathrm{M}_{\mathrm{ML}}$ and the $\mathrm{M}_{\mathrm{A}}$ components. These were calculated according to (1) and (2).

$$
\begin{aligned}
& M_{M L}=M_{M L L C}+d F_{A} \\
& M_{A}=M_{A L C}+d F_{M L}
\end{aligned}
$$

The naming convention used for forces and moments

\begin{tabular}{|c|c|c|}
\hline Distal end of Fixture & Load Cell & Description \\
\hline $\mathrm{F}_{\mathrm{ML}}$ & $F_{M L ~ L C}$ & Medio-lateral force \\
\hline $\mathrm{F}_{\mathrm{A}}$ & $\mathrm{F}_{\mathrm{ALC}}$ & Anterior-posterior force \\
\hline $\mathrm{F}_{\mathrm{L}}$ & $F_{L L C}$ & Longitudinal force \\
\hline$F_{\text {Res }}$ & $F_{\text {Res LC }}$ & Resultant force \\
\hline $\mathrm{M}_{\mathrm{ML}}$ & MML LC & Medio-lateral moment \\
\hline $\mathrm{M}_{\mathrm{A}}$ & $\mathrm{M}_{\mathrm{ALC}}$ & Anterior moment \\
\hline $\mathrm{M}_{\mathrm{L}}$ & $\mathrm{M}_{\mathrm{L} L C}$ & Longitudinal moment \\
\hline $\mathrm{M}_{\mathrm{B}}$ & $\mathrm{MB}_{\mathrm{B} C \mathrm{C}}$ & Bending moment \\
\hline MRes & $M_{\text {Res LC }}$ & Resultant moment \\
\hline
\end{tabular}
throughout this study are presented in Table II.

For each subject and activity, the load data was divided into gait cycles by detection of onset of longitudinal loading $\left(\mathrm{F}_{\mathrm{L}}\right.$ component). To avoid inclusion of transitory movements in the analysis of each activity, incomplete gait cycles at the beginning and at the end of each trial were excluded from the analysis. Maximum values (largest magnitude) of each variable in Table II were extracted for each cycle. These values were subsequently used to determine overall maximum, and mean values (of cycle maximums) per activity for each subject.

Walking on level ground was more deeply analyzed in order to characterize the exact load states at the instances of peak loading (peak loading defined as maximum stress-state rather than maximum resultant force). This was done to determine the direction and magnitude of the resultant force and resultant moment vectors at the instances corresponding to the highest stress-state, and to compare across subjects to obtain data which could potentially be used as input for development of a clinically relevant, standardized test method for bone-anchored prostheses for individuals with transfemoral amputation.

Given the magnitudes of the force and moment components and the fact that both the residual femur and the implant are principally cylindrical in shape, the maximum stress state is dominated by the contribution from the bending moment $\mathrm{M}_{\mathrm{B}}$. Thus, the bone-anchored implant and the bone are subjected to maximum stress at the instances of peak bending moment. Therefore, instances of the peak bending moments in the first and second half of the stance phase (presumably corresponding to heel strike and toe-off) were identified (using custom Matlab script) for each gait cycle, and the resultant force and moment vectors were calculated at each of these instances. Mean vectors were then calculated across gait cycles for each subject including the deviation from the longitudinal axis of the boneanchored implant.

\section{Statistical analysis}

We explored whether there were relationships between the activity performed and the peak magnitudes of measured forces and moments. For each activity, peak forces and moments were pair-wise compared with corresponding values for level walking (reference activity) for each subject completing both activities. Each data set was checked for normality using the Shapiro-Wilk test. To test the HO hypothesis, stating that the difference in peak force (or peak moment) for any activity with respect to level walking has a zero median, tests for significance were performed using two-tailed t-tests for datasets that were considered sufficiently normally distributed ( $\mathrm{p}$-value higher than 0.7 for Shapiro-Wilk test) and two-tailed Wilcoxon signed rank tests were used for the remainder of the data sets. To investigate whether the use of a crutch in the contralateral hand of the side of amputation had a significant effect on the load level, the same procedure was performed but with the data set for corresponding activity performed without a crutch as reference. Since none of the subjects performed stair ascent and stair descent both with and without a crutch, this statistical investigation was not done for the stair activities. The results are presented in Fig. 2. The data from Fig 2 a) and c) is available in tabulated form as supplementary material. 
(a)

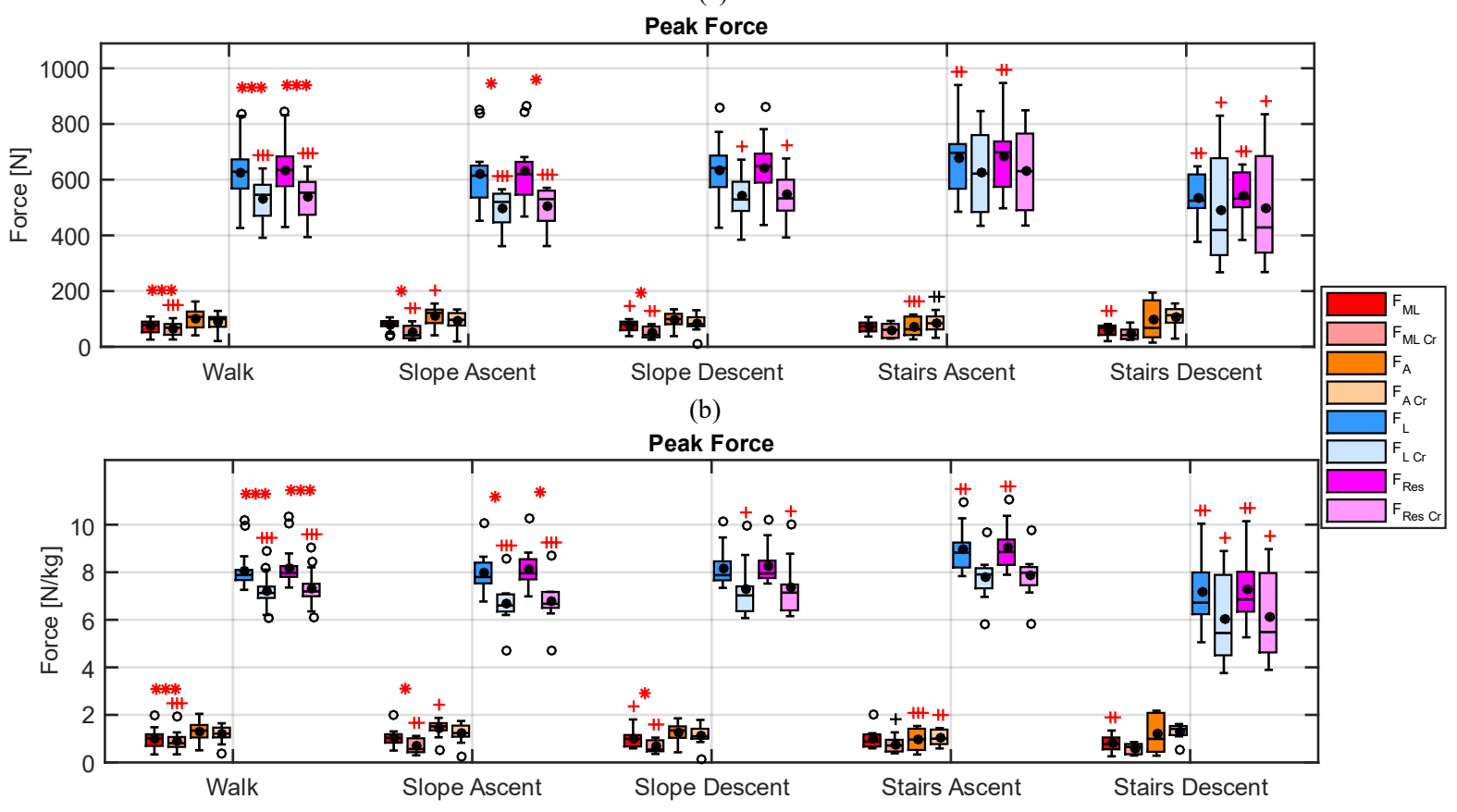

(c)

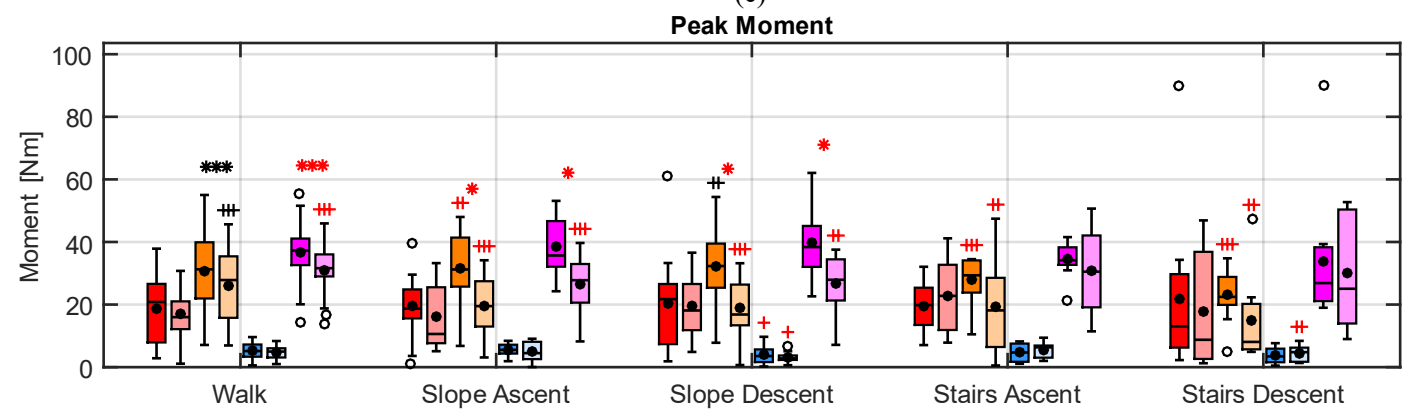

(d)

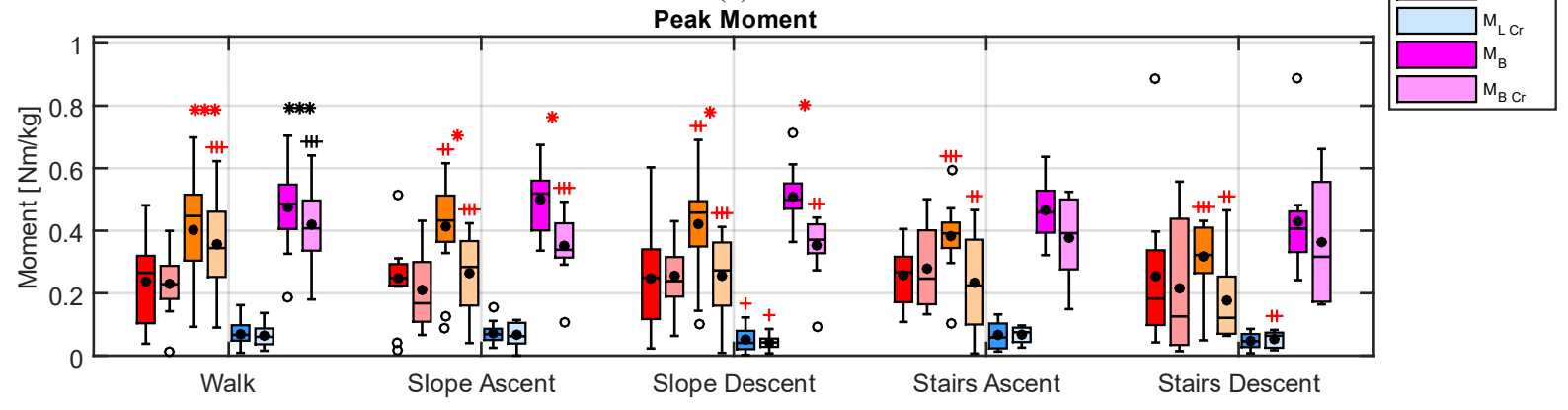

Fig. 2. (a) Peak force (magnitude) in Newtons for each activity. (b) Peak force (magnitude) in Newtons per kg body mass. (c) Peak moment (magnitude) in Nm for each activity. (d) Peak moment (magnitude) in Nm per kg body mass. The data represents the mean peak magnitude of force/moment of all gait cycles per participant and activity. Mean values across participants are indicated with a solid black dot. Asterisks indicate significant difference between the force level when walking with or without a crutch with the number of asterisks representing alpha levels $0.05,0.01$, and 0.001 respectively. Plus signs indicate significant difference between the force level for the activity compared with level walking (reference activity). The number of plus signs represents alpha levels $0.05,0.01$, and 0.001 respectively. Black color of asterisk or plus sign indicate statistically significant difference determined from two-tailed t-tests. Red color indicates statistically significant difference determined from two-tailed Wilcoxon signed rank tests.

\section{RESULTS}

\section{A. Maximum load state during daily activities}

The longitudinal force component $\left(\mathrm{F}_{\mathrm{L}}\right)$ dominates the measured peak forces in all activities, thus the resultant force $\left(F_{\text {Res }}\right)$ closely follows $F_{L}$. The mean resultant peak forces ranged from 268-947 $\mathrm{N}$ across activities and participants. The mean peak resultant force per activity ranged from $498 \mathrm{~N}$ (stairs descent with crutch) to $684 \mathrm{~N}$ (stairs ascent). Compared with 
level walking, statistically significant different peak forces were found for $\mathrm{F}_{\mathrm{A}}$ at slope and stairs ascent, $\mathrm{F}_{\mathrm{ML}}$ at slope and stairs descent, and $F_{L}$ and $F_{R e s}$ at stair ascent and stairs descent. When normalizing the forces to Newtons per $\mathrm{kg}$ body mass, statistically significant differences were found for the same force components and activities (Fig. 2 (b)). Of the studied activities, stair ascent generated the highest resultant forces (mean $9.1 \mathrm{~N} / \mathrm{kg}$ ) while stair descent generated the lowest (mean $7.3 \mathrm{~N} / \mathrm{kg})$.

For the moment loads, $\mathrm{M}_{\mathrm{A}}$ and $\mathrm{M}_{\mathrm{ML}}$, which give rise to the bending moment, were the dominant components for all activities. The mean peak bending moment ranged from $7.1 \mathrm{Nm}$ and $90.1 \mathrm{Nm}$ across activities and subjects. However, the 90 $\mathrm{Nm}$ bending moment, which was measured for a single subject during stair descent, was far above any other measured bending moments and can be regarded as an outlier. The mean peak bending moment per activity ranged from $26.5 \mathrm{Nm}$ (slope ascent with crutch) to $39.8 \mathrm{Nm}$ (slope descent). The measured peak longitudinal moments, $\mathrm{M}_{\mathrm{L}}$, ranged from $0 \mathrm{Nm}$ to $9.7 \mathrm{Nm}$ across activities and subjects. The mean peak longitudinal moment per activity ranged from $3.1 \mathrm{Nm}$ (slope descent with crutch) to $5.5 \mathrm{Nm}$ (stairs ascent with crutch). Compared with level walking, statistically significant differences were found for the $\mathrm{M}_{\mathrm{A}}$ component during slope and stairs ascent and descent, and for $\mathrm{M}_{\mathrm{L}}$ during slope descent. These statistically significant differences remained also when the moments were normalized to $\mathrm{Nm}$ per $\mathrm{kg}$ body mass.

\section{B. The influence of walking with a walking aid}

The use of a crutch reduced the peak resultant forces with averages ranging from $5.5 \%$ (slope descent (effect size 1.17)) to $12.6 \%$ (slope ascent (effect size 6.03)). The peak bending moments were reduced with averages ranging from $13.2 \%$ (level walking (effect size 2.42)) to $15.6 \%$ (slope ascent (effect size 3.73)).

\section{Effect of different strategies for stair ascent/descent}

A large variability in walking strategy between participants was noted especially for stair ascent and descent. Stair ascent is known to be difficult for individuals with transfemoral amputation due to the inability to generate net power output across the knee joint on the amputated side to lift the body upward. This inability leads to that most individuals with transfemoral amputation have an altered strategy where they first take a step up with their nonaffected limb and then follow to the same step in the staircase with their prosthetic leg. In

\section{TABLE III}

\begin{tabular}{ll}
\hline Name & Description \\
\hline StUp H1P1 & $\begin{array}{l}\text { Stair ascent, nonaffected limb leading, prosthetic foot following to } \\
\text { same step }\end{array}$ \\
StUpCr H1P1 & $\begin{array}{l}\text { Stair ascent with crutch, nonaffected limb leading, prosthetic foot } \\
\text { following to same step }\end{array}$ \\
StUp H2P2 & $\begin{array}{l}\text { Stair ascent, nonaffected limb leading with two steps prosthetic } \\
\text { foot following to same step }\end{array}$ \\
StDn P1H1 & $\begin{array}{l}\text { Stair descent, prosthetic limb leading, nonaffected foot following to } \\
\text { same step }\end{array}$ \\
StDnCr P1H1 & $\begin{array}{l}\text { Stair descent with crutch, prosthetic limb leading, nonaffected foot } \\
\text { following to same step }\end{array}$ \\
StDn P1H2 & $\begin{array}{l}\text { Stair descent, reciprocal gait } \\
\text { Stair descent with crutch, reciprocal gait }\end{array}$
\end{tabular}
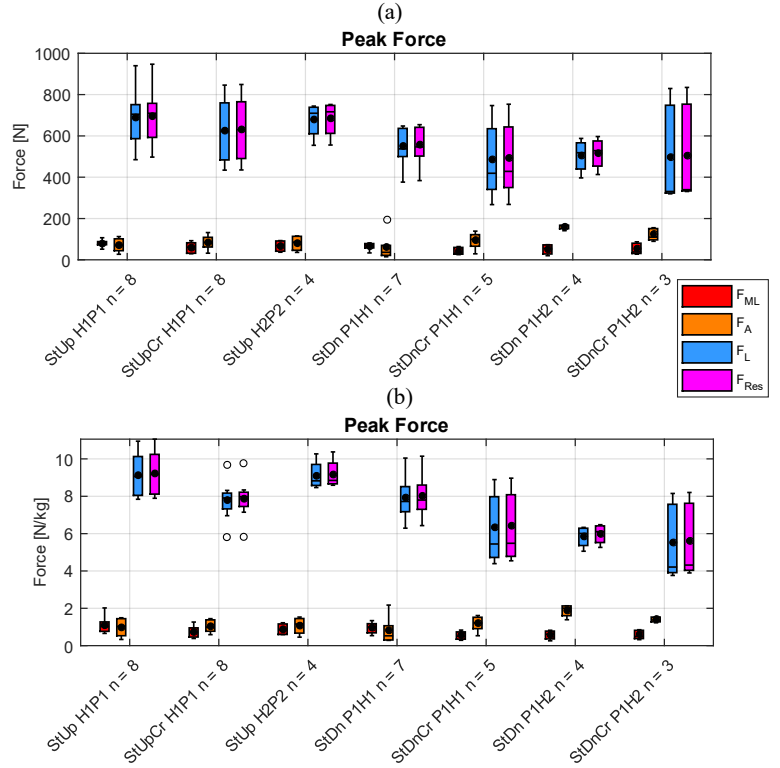

(c)

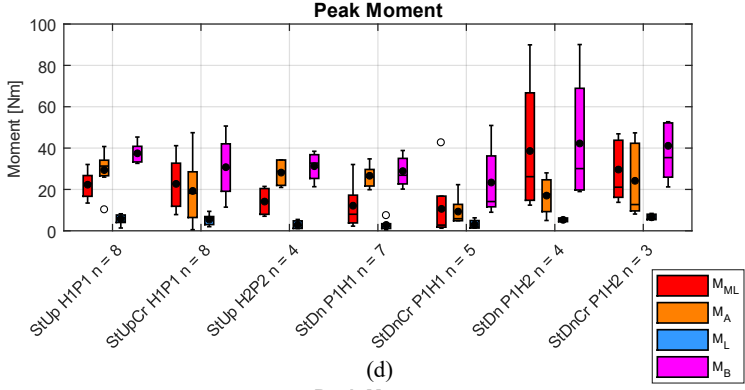

Peak Moment

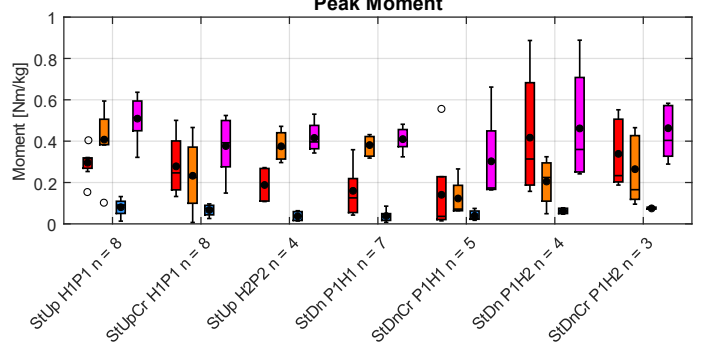

Fig. 3. (a) Peak force (magnitude) in Newtons for each activity. (b) Peak force (magnitude) in Newtons per $\mathrm{kg}$ body mass. (c) Peak moment (magnitude) in $\mathrm{Nm}$ for each activity. (d) Peak moment (magnitude) in $\mathrm{Nm}$ per $\mathrm{kg}$ body mass. The data represents the average peak magnitude of force/moment of all gait cycles per patient and activity. Mean values are indicated with a solid black dot.

order to increase the speed of ascent, some subjects prefer to take two steps at a time with their nonaffected limb and then follow to the same step with the prosthesis. Both of these strategies were common among the subjects in the current study. Several strategies were also observed for stair descent. Seven subjects used reciprocal stair decent, while twelve subjects instead used a more conservative single step descent approach with the prosthetic leg leading and the nonaffected limb following to the same step. Although many modern prosthetic knee joints have a built in yielding function (all MPKs and Mauch knee have full yielding function and 3R60 has partial yielding function, see Table I) which prevent inadvertent collapse during stair descent, a single step descent provides an additional level of security, and is thus preferred by 
many individuals with transfemoral amputation. The boxes representing the stairs data in Fig. 2 present an aggregate of these different strategies for stair ascent and descent. Fig. 3 presents the same data for stair ascent/descent but separated per ascent/descent strategy. The data is available in tabulated form as supplementary material. The strategies are named according to Table III.

\section{Resultant loading directions at peak stress states}

For the level walking activity, the load data was more thoroughly evaluated. The resultant force and moment vectors at the first and second stress peak are presented in Fig. 4. The

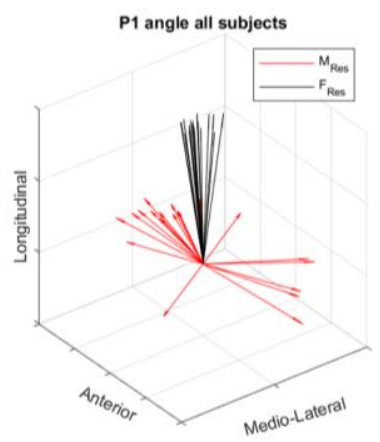

(a)

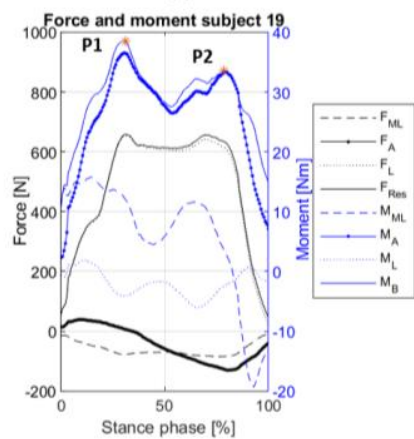

(c)

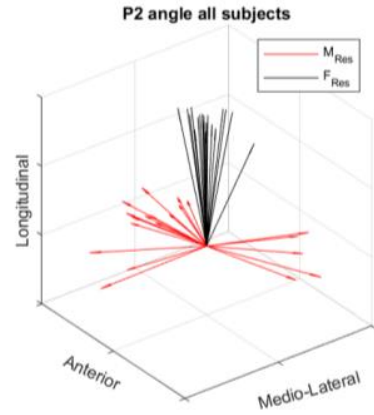

(b)

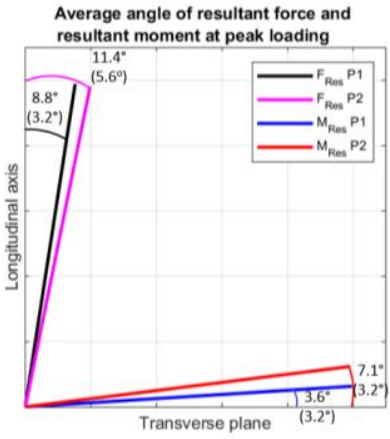

(d)
Fig 4 . a) and b), Mean direction of resultant force and resultant moment vectors for each subject at the first (P1) and second (P2) stress peaks during level walking. c) All force and moment components during the stance phase of a single subject. d) Across subjects average deviation from the longitudinal axis and the transverse plane in degrees (standard deviation in parentheses) for the resultant force and resultant moments at the instances of P1 and P2 respectively.

resultant force had a mean deviation of $8.8^{\circ}$ and $11.4^{\circ}$ from the longitudinal axis and the resultant moment had a mean deviation of $3.6^{\circ}$ and $7.1^{\circ}$ from the transverse plane for the first and second stress peaks, respectively. The mean longitudinal moments were $2.4 \mathrm{Nm}$ and $4.3 \mathrm{Nm}$ at the first and second stress peaks, respectively.

\section{DISCUSSION}

To assess how the measured peak forces and moments compare with earlier work, a comparison was made with four previous studies [16]-[19], however it must be noted that the study samples in the two studies by Lee et al. [17], [18] is largely overlapping. Differences in test conditions (step height and the slope angle), reported load variables, number of subjects, and analyzed activities between the studies imposes limitations on the comparisons. Especially large differences were noted for the height of the steps in the stairs where previous studies have reported $30 \mathrm{~cm}$ high steps compared with $15.5 \mathrm{~cm}$ in the present study. A smaller relative difference was noted for the slope angle $\left(6.5^{\circ}\right.$ in previous studies and $7.3^{\circ}$ in the present study). For level walking, the test conditions can be regarded as similar between the studies. The results from the comparison is presented in Table IV.

The mean peak forces and moments in the present study were within the range of forces and moments that have been reported in prior studies [16]-[19], as indicated in Table IV. The comparisons are most valid for the level walking activity where the test conditions were similar. However, differences in the sample population should not be ignored when interpreting the results. Concerning activities other than walking, Lee et al., provides the best benchmark to the present study since it contains multiple subjects and has a comparable, albeit slightly higher, mean weight in the sample population [17]. When analyzing how the loads in their study compared to our present study, the only apparent trend consistent across all activities is the higher longitudinal forces they found, which to a large

TABLE IV

\begin{tabular}{|c|c|c|c|c|c|}
\hline \multicolumn{2}{|l|}{ Study } & Lee 2007 & Lee 2008 & $\begin{array}{c}\text { Frossard } \\
2013\end{array}$ & $\begin{array}{l}\text { Stenlund } \\
2016\end{array}$ \\
\hline \multirow{3}{*}{\multicolumn{2}{|c|}{$\begin{array}{l}\text { Number of subjects } \\
\text { Mean weight [kg] (St.d.) } \\
\text { Mean age [years] (St.d.) }\end{array}$}} & 9 & 12 & 1 & 5 \\
\hline & & $82.5(16.8)$ & $84.3(16.8)$ & $96.5(\mathrm{n} / \mathrm{a})$ & $73.5(15.3)$ \\
\hline & & $47(9.7)$ & $48(9.7)$ & $41(n / a)$ & $58.8(15.5)$ \\
\hline \multicolumn{2}{|r|}{ Variable } & \multicolumn{4}{|c|}{ Difference compared with present study [\%] } \\
\hline \multirow{5}{*}{ Level Walking } & $\mathrm{F}_{\mathrm{L}}$ & +8.0 & +11.7 & +5.2 & -10.6 \\
\hline & $\mathrm{M}_{\mathrm{ALC}}$ & -16.9 & -16 & -64 & - \\
\hline & MMLLC & +7.2 & +10 & -17.6 & - \\
\hline & $M_{L}$ & -5.2 & - & -35 & - \\
\hline & $\mathrm{M}_{\mathrm{B}}$ & - & - & - & +5.6 \\
\hline \multirow{4}{*}{ Slope Ascent } & $\mathrm{F}_{\mathrm{L}}$ & +13.4 & - & +9.4 & - \\
\hline & $\mathrm{M}_{\mathrm{ALC}}$ & -9.3 & - & -61.2 & - \\
\hline & M MLLC & -18.4 & - & -28.1 & - \\
\hline & $\mathrm{M}_{\mathrm{L}}$ & +6.3 & - & -13.5 & - \\
\hline \multirow{4}{*}{ Slope Descent } & $\mathrm{FL}_{\mathrm{L}}$ & +10.2 & - & -0.7 & - \\
\hline & $\mathrm{M}_{\mathrm{ALC}}$ & -4.8 & - & -66.2 & - \\
\hline & $M_{M L L C}$ & +51 & - & +55.6 & - \\
\hline & $\mathrm{M}_{\mathrm{L}}$ & +31.6 & - & -32.1 & - \\
\hline \multirow{4}{*}{ Stairs Ascent } & $\mathrm{F}_{\mathrm{L}}$ & +12.1 & - & +7.7 & - \\
\hline & $\mathrm{M}_{\mathrm{ALC}}$ & -3.7 & - & -83.0 & - \\
\hline & $M_{M L L C}$ & -58.5 & - & -69.2 & - \\
\hline & $M_{L}$ & -22.8 & - & -33.2 & - \\
\hline \multirow{4}{*}{ Stairs Descent } & $\mathrm{F}_{\mathrm{L}}$ & +21.4 & - & -1.3 & - \\
\hline & $\mathrm{M}_{\mathrm{ALC}}$ & +3.0 & - & -69 & - \\
\hline & MMLLC & -8.9 & - & -14.5 & - \\
\hline & $\mathrm{ML}_{\mathrm{L}}$ & +39.7 & - & -42.1 & - \\
\hline
\end{tabular}

TABLE IV. Comparison with load data from previous studies. The peak forces and moments were normalized to bodyweight (BW) and then compared as mean values across the subjects of respective study. Lee 2007 did not present the values as \% BW and therefore comparison with this study was made with nominal values. The values are presented as relative difference compared with the present study; thus a positive value indicate that the load level in the previous study was higher than corresponding value for the present study. A "“-" indicates that a comparison could not be made due to lack of information.

extent can be attributed to the slightly heavier sample population. Notably, the large difference in the stairs step height between the studies did not lead to any conclusive differences in the mean peak moments between the studies. Overall, it can be concluded that the load measurements obtained in this study are in line with previously reported results. 
There was an extreme outlier for the $\mathrm{M}_{\mathrm{ML}}$ component and consequently for the bending moment $\mathrm{M}_{\mathrm{B}}$ for a single subject during stairs decent. The mean peak value of $\mathrm{M}_{\mathrm{B}}$ for this subject was $90.1 \mathrm{Nm}$ during stairs descent, whereas the mean peak values for the remaining subjects performing the same activity was merely $31.4 \mathrm{Nm}$. The subject who exhibited these extreme bending moments was one the four subjects who used reciprocal gait without a crutch during stair decent, which intuitively is the highest loading descent strategy of the ones observed in the study. Furthermore, he was one of the heaviest subjects in the study $(101 \mathrm{~kg})$ and more than $15 \mathrm{~kg}$ heavier than any of the three other subjects using the same stair decent strategy. This subject also recorded the highest mean peak bending moments for slope descent $(62.1 \mathrm{Nm})$, but apart from those results, he did not stand out with exceptional load levels. Except for this outlier, none of the activities generated mean peak resultant forces or mean peak bending moments that were dramatically higher than corresponding values for level walking. For the mean peak resultant forces, significant differences compared with level walking were found for stairs ascent, (higher, $\mathrm{p}=0.002$ ), and stairs descent, (lower, $\mathrm{p}=$ 0.0049). The reason why stair ascent generated higher forces than level walking might be related to the fact that during stair ascent an additional ground reaction force is needed to raise the center of gravity of the body upwards, whereas for stair descent the situation is reversed. However, these differences could also result from partial loading of the handrail during each of the stairs activities. Since the forces in the handrail were not quantified, no conclusions regarding this can be drawn. Moreover, most subjects did not use a reciprocal walking strategy when walking on the stairs, thus leading to reduced loading compared with conventional stair ascent/descent, especially since individuals with transfemoral amputation, are generally leading with the intact limb during stair ascent and the prosthesis during stair descent. No significant differences were found for the mean peak bending moment in any of the activities compared with level walking.

Using a crutch in one hand reduced both the load levels and the moment levels. However, the reductions were only moderate, with slope ascent being the activity that presented the largest average reductions both for the resultant force $(12.6 \%)$ and the bending moment $(15.6 \%)$. For level walking the average reductions were $9.7 \%$ and $13.2 \%$ for the resultant force and the bending moments respectively. This can be compared with the single-subject case study [21] which reported a reduction of $15 \%$ of the peak longitudinal force when a single crutch was used.

If the original peak loads generate stresses in the bone or the bone-anchored implant that are above fatigue inducing stress levels, even a small increase of the load could lead to a substantial reduction in fatigue life. Conversely, a minor reduction in the peak loads (for example by the use of a crutch) could lead to substantial improvements in fatigue life. Moreover, for the case of bone tissue, the fatigue damage process could be counteracted to a certain extent by remodeling and tissue repair processes.

Level walking did not exhibit substantially lower load levels than the other activities for most subjects, and since this is the activity which generates the highest number of load cycles, it is reasonable to believe that this activity alone would contribute to the majority of the sustained fatigue damage to the implant system and the bone. This is under the assumption that no, or very little, damage is sustained during any activity other than those examined in this study. A standardized method for structural testing of bone-anchored implant systems should therefore try to mimic the load characteristics associated with level walking. The load levels presented in Fig. 2 and the angles presented in Fig. 4 could aid in this process.

\section{LIMITATIONS}

The objective of the study was to determine the maximum load exposure of the bone-anchored implant system during activities of daily living, which required that each subject used their own prosthetic components and performed the activities in the same way that they normally do. This provided us with clinically relevant load measurements. However, it made it challenging to objectively compare the measured load data between activities, since different strategies (stair ascent/descent) and different extent of walking aids were used. This is the largest limitation in this study. A related limitation is the small sample size, which limits the possibility to draw strong conclusions for a larger population. Nevertheless, the subjects in our sample varied widely in terms of body weight $(53 \mathrm{~kg}-102 \mathrm{~kg})$ and age $(26-73)$, and therefore covered most of the eligible population in terms of these factors.

\section{CONCLUSION}

Based on the load measurements, no activity could be singled out as generating particularly high loads on the implant for the majority of the participants. With reference to level walking, no activity generated statistically significantly higher bending moments on the implant, and only stair ascent generated statistically significantly higher resultant forces. The results indicate that the loading during level walking is the most important driver of fatigue damage. However, the small sample size, and large inter-subject variations limit the possibility to draw general conclusions for this cohort. As observed in this study, individual subjects can generate bending moments as high as $90 \mathrm{Nm}$ during stair descent. For these subjects a more conservative stair descent strategy, or the use of a walking aid is recommended in order to reduce the loading.

On a subject level, the load measurements from this study can serve as a guide for individual recommendations in terms of walking strategy and extent of walking aid usage. In combination, with load measurements from larger population samples the obtained load data can contribute to more general guidelines for this cohort. Our results can also be used as design input for further developments of bone-anchored implant systems for prosthetic attachment of limb prostheses along with their associated safety devices, as these indicate minimum 
requirements regarding cyclic load exposure as well as adequate safety release levels.

\section{REFERENCES}

N. L. Dudek, M. B. Marks, S. C. Marshall, and J. P. Chardon, "Dermatologic conditions associated with use of a lower-extremity prosthesis," Arch. Phys. Med. Rehabil., vol. 86, no. 4, pp. 659-663, 2005.

F. T. Hoaglund, H. E. Jergesen, L. Wilson, L. W. Lamoreux, and R. Roberts, "Evaluation of problems and needs of veteran lower-limb amputees in the San Francisco Bay Area during the period 19771980.,"J. Rehabil. R\&D, 1983.

[3] H. E. J. Meulenbelt, J. H. B. Geertzen, M. F. Jonkman, and P. U. Dijkstra, "Skin problems of the stump in lower limb amputees: 1. A clinical study," Acta Derm. Venereol., 2011.

R. Tranberg, R. Zügner, and J. Kärrholm, "Improvements in hipand pelvic motion for patients with osseointegrated trans-femoral prostheses," Gait Posture, vol. 33, no. 2, pp. 165-168, 2011.

L. Paternò, M. Ibrahimi, E. Gruppioni, A. Menciassi, and L. Ricotti, "Sockets for limb prostheses: A review of existing technologies and open challenges," IEEE Trans. Biomed. Eng., vol. 65, no. 9, pp. 1996-2010, 2018.

[6] R. Brånemark, P.-I. Brånemark, B. Rydevik, and R. R. Myers, "Osseointegration in skeletal reconstruction and rehabilitation," $J$. Rehabil. Res. Dev., vol. 38, pp. 175-181, 2001.

[7] A. Thesleff, R. Brånemark, B. Håkansson, and M. Ortiz-Catalan, "Biomechanical Characterisation of Bone-anchored Implant Systems for Amputation Limb Prostheses: A Systematic Review," Annals of Biomedical Engineering, pp. 1-15, 2018.

E. Häggström, K. Hagberg, B. Rydevik, and R. Brånemark, "Vibrotactile evaluation: osseointegrated versus socket-suspended transfemoral prostheses.," J. Rehabil. Res. Dev., vol. 50, no. 10, pp. 1423-34, 2013.

R. Jacobs, R. Brånemark, K. Olmarker, B. Rydevik, D. Van Steenberghe, and P. I. Brånemark, "Evaluation of the psychophysical detection threshold level for vibrotactile and pressure stimulation of prosthetic limbs using bone anchorage or soft tissue support.," Prosthet. Orthot. Int., vol. 24, no. 2, pp. 133$142,2000$.

[10] K. Hagberg, E. Hansson, and R. Brånemark, "Outcome of percutaneous osseointegrated prostheses for patients with unilateral transfemoral amputation at two-year follow-up," Arch. Phys. Med. Rehabil., vol. 95, no. 11, pp. 2120-2127, 2014.

[11] R. P. Brånemark, K. Hagberg, K. Kulbacka-Ortiz, Ö. Berlin, and B. Rydevik, "Osseointegrated Percutaneous Prosthetic System for the Treatment of Patients With Transfemoral Amputation," J. Am. Acad. Orthop. Surg., vol. 00, no. 00, p. 1, 2018.
K. Hagberg and R. Brånemark, "One hundred patients treated with osseointegrated transfemoral amputation prostheses -Rehabilitation perspective," J. Rehabil. Res. Dev., vol. 46, no. 3, pp. 331-344, 2009.

[13] K. Hagberg, S. A. Ghassemi Jahani, K. Kulbacka-Ortiz, P. Thomsen, H. Malchau, and C. Reinholdt, "A 15-year follow-up of transfemoral amputees with bone-anchored transcutaneous prostheses,” Bone Joint J., vol. 102-B, no. 1, pp. 55-63, 2020.

[14] L. A. Frossard, R. Tranberg, E. Häggstrom, M. Pearcy, and R. Brånemark, "Load on osseointegrated fixation of a transfemoral amputee during a fall: Loading, descent, impact and recovery analysis," Prosthet. Orthot. Int., 2010.

[15] L. Frossard et al., "Monitoring of the load regime applied on the osseointegrated fixation of a trans-femoral amputee: A tool for evidence-based practice," Prosthet. Orthot. Int., vol. 32, no. 1, pp. 68-78, 2008.

[16] L. Frossard, E. Häggström, K. Hagberg, and R. Brånemark, "Load applied on bone-anchored transfemoral prosthesis: characterization of a prosthesis-a pilot study.," J. Rehabil. Res. Dev., vol. 50, no. 5, pp. 619-34, 2013.

[17] W. C. C. Lee et al., "Kinetics of transfemoral amputees with osseointegrated fixation performing common activities of daily living," Clin. Biomech., vol. 22, no. 6, pp. 665-673, 2007.

[18] W. C. C. Lee et al., "Magnitude and variability of loading on the osseointegrated implant of transfemoral amputees during walking," Med. Eng. Phys., vol. 30, no. 7, pp. 825-833, 2008.

[19] P. Stenlund, M. Trobos, J. Lausmaa, R. Brånemark, P. Thomsen, and A. Palmquist, "The effect of load on the bone-anchored amputation prostheses," J. Orthop. Res., vol. 35, no. 5, pp. 1113$1122,2016$.

[20] L. Frossard, "Loading characteristics data applied on osseointegrated implant by transfemoral bone-anchored prostheses fitted with basic components during daily activities," Data Br., 2019.

[21] L. Frossard, K. Hagberg, E. Häggström, and R. Brånemark, "Loadrelief of walking aids on osseointegrated fixation: Instrument for evidence-based practice," IEEE Trans. Neural Syst. Rehabil. Eng., vol. 17, no. 1, pp. 9-14, 2009.

[22] S. R. Koehler, Y. Y. Dhaher, and A. H. Hansen, "Cross-validation of a portable, six-degree-of-freedom load cell for use in lower-limb prosthetics research," J. Biomech., vol. 47, no. 6, pp. 1542-1547, 2014

[23] S. R. Koehler-McNicholas, R. D. Lipschutz, and S. A. Gard, "The biomechanical response of persons with transfemoral amputation to variations in prosthetic knee alignment during level walking," $J$. Rehabil. Res. Dev., 2017.

[24] L. Frossard, B. Leech, and M. Pitkin, "Automated characterization of anthropomorphicity of prosthetic feet fitted to bone-anchored transtibial prosthesis," IEEE Trans. Biomed. Eng., 2019.

[25] L. Frossard, B. Leech, and M. Pitkin, "Inter-participant variability data in characterization of anthropomorphicity of prosthetic feet fitted to bone-anchored transtibial prosthesis," Data Br., 2019. 